1 Hacettepe Journal of Mathematics and Statistics

h Volume 47 (2) (2018), 331-337

\title{
Abelian product of free Abelian and free Lie algebras
}

\author{
Zeynep Özkurt * and Naime Ekici ${ }^{\dagger}$
}

\begin{abstract}
Let $F_{n}$ be a free Lie Algebra of finite rank $n$ and $A$ be a free abelian Lie algebra of finite rank $m \geq 0$. We investigate the properties of the generating sets and subalgebras of the abelian product $A *_{a b} F_{n}$. Moreover these properties are used to solve the membership problem for $A *_{a b} F_{n}$.
\end{abstract}

Keywords: Generating set, Abelian product, Free Lie Algebra

2000 AMS Classification: 17B01

Received: 12.12.2014 Accepted: 08.02.2017 Doi : 10.15672/HJMS.2017.455

\section{Introduction}

Let $F_{n}$ be a free Lie algebra with a free generating set $X=\left\{x_{1}, x_{2}, \ldots, x_{n}\right\}$ over a field of $K$ of characteristic zero and $A$ be a free abelian Lie algebra generated by a set $Y=\left\{y_{1}, \ldots, y_{m}\right\}$ over $K$. Let $D$ be the cartesian subalgebra of the free product $A * F_{n}$ of $A$ and $F_{n}$, that is ,the kernel of the canonical homomorphism from $A * F_{n}$ onto the direct sum $A \oplus F_{n}$. The $k-t h$ solvable product of $A$ and $F_{n}$ is defined as $\left(A * F_{n}\right) / \delta^{k}\left(A * F_{n}\right) \cap D$, where $\delta^{k}\left(A * F_{n}\right)$ is the $k-t h$ term of the derived series of $A * F_{n}$. In the case $k=0$ the $k-t h$ solvable product becomes $\left(A * F_{n}\right) / D$, which is isomorphic to the direct sum $A \oplus F_{n}$. However for the sake of compliance with the sprit of our work we shall refer to $0-t h$ solvable product of $A$ and $F_{n}$ as abelian product and we denote it by $A *_{a b} F_{n}$. Free abelian Lie algebras and free Lie algebras have been extensively studied in the literature. Many questions that admit simple solutions when dealing with $A$ and $F_{n}$ individually, require far more involved solutions over abelian product $A *_{a b} F_{n}$ of $A$ and $F_{n}$. This is the case, when one considers subalgebras and generating sets: Generating sets of $A *_{a b} F_{n}$ have common properties with generating sets of free abelian and free Lie algebras. It is well known that every subalgebra of a free abelian Lie algebra is free abelian and every subalgebra of free Lie algebra is again free. These two facts lead to the same property

*Çukurova University, Department of Mathematics, 01330, Adana ,Turkey, Email: zyapti@cu.edu.tr

${ }^{\dagger}$ Çukurova University, Department of Mathematics, 01330, Adana ,Turkey, Email: nekici@cu.edu.tr 
for abelian product $A *_{a b} F_{n}$. As another example we may consider the study of test elements and test sets. The Lie algebra $A *_{a b} F_{n}$ doesn't have test elements but in the free Lie algebra $F_{n}$ there are many test elements. Interest in the test ranks of the abelian product $A *_{a b} F_{n}$ is explained in [4]. In $[5,6]$ test sets and test ranks of solvable and metabelian products of groups were studied.

In this paper we investigate subalgebras of abelian products of the form $A *_{a b} F_{n}$ and we define there several properties shared by both families of free abelian and free Lie algebras. As a consequence we observe that the Lie algebras of the form $A *_{a b} F_{n}$ have solvable membership problem. The motivation of this work is based on the studies in groups. We use the methods introduced in [3] to prove our results.

For any nonempty subset $Z$ of a Lie algebra by $\langle Z\rangle$ we mean the subalgebra generated by $Z$. For the rank of any free Lie algebra $B$ we write $\operatorname{rank}(B)$.

\section{Abelian Product of $A$ and $F_{n}$}

Let $X=\left\{x_{1}, \ldots, x_{n}\right\}$ and $Y=\left\{y_{1}, \ldots, y_{m}\right\}$ be disjoint sets, where $m, n \geqslant 0$. The ideas of this section are similar to the corresponding ideas in group theory [3], but have subtle differences. Consider the Lie algebra $L$ defined by the presentation

$$
L=<Y \cup X:\left[y_{i}, y_{j}\right],\left[y_{i}, x_{s}\right], 1 \leq i, j \leq m, 1 \leq s \leq n, y_{i} \in Y, x_{s} \in X>.
$$

Let $A$ and $F_{n}$ be the subalgebras of $L$ generated, respectively, by $Y$ and $X$. We shall refer to $A=<Y>$ and $F_{n}=<X>$ as the free-abelian and free parts of $L$, respectively. It is clear that the Lie algebra $L$ is the abelian product of $A$ and $F_{n}$, namely $A *_{a b} F_{n}$ .Therefore every element $g$ of $L$ can be written as $g=u+v$ in a unique way, where $u \in A, v \in F_{n}$. Naturally we can write $g$ as $\sum \alpha_{i} y_{i}+v$ where $\alpha_{i} \in K, y_{i} \in Y, v \in F_{n}$. By straightforward computations we see that the center of $L$ is $A$.

2.1. Definition. Let $(Z, T)$ be a pair of subsets of $L$. If

i) $Z$ is an abelian basis of the center of $L$,

ii) $T$ is a free generating set of a free subalgebra of $L$,

iii) $L=<Z \cup T>$,

then the pair $(Z, T)$ of subsets of $L$ is called a minimal generating pair of $L$. In this case we shall say that $Z \cup T$ is a minimal generating set of $L$.

Let $(Z, T)$ be a minimal generating pair of $L$. From definition it follows that $\langle Z\rangle$ $\cap<T\rangle=\{0\}$ and $Z \cap T=\emptyset$, since $\langle Z>\cap\langle T\rangle$ is contained in the center of $L$, but no nontrivial element of $\langle T\rangle$ belongs to it. Since we can consider the set $Z$ as the elements in $Z \cup T$ which belong to the center of $L$, and $T$ as the remaining elements then the set $Z \cup T$ is linearly independent modulo the derived subalgebra of $L$. So $L$ cannot be generated by less than $|Z|+|T|$ elements, where $|Z|$ and $|T|$ are cardinalities of $Z$ and $T$, respectively. Therefore the set $Z \cup T$ is a minimal generating set of $L$ defined by the minimal generating pair $(Z, T)$. We shall refer to $|Z|+|T|$ as the rank of $L$ and we denote it by $\operatorname{rank}(L)$.

The following lemma shows that the ranks of the free abelian and free parts of $L$ are invariants of $L$.

2.2. Lemma. Let $A, B$ be free abelian Lie algebras and $F$ and $G$ be free Lie algebras of ranks at least 2. Then the Lie algebra $A *_{a b} F$ is isomorphic to $B *_{a b} G$ if and only if $\operatorname{rank}(A)=\operatorname{rank}(B)$ and $\operatorname{rank}(F)=\operatorname{rank}(G)$

The proof of Lemma 2.2 is straightforward. So we omit it.

2.3. Corollary. Every abelian product $M$ of a nonzero free abelian Lie algebra with a free Lie algebra has a minimal generating pair. Moreover, every minimal generating pair 
$(Z, T)$ satisfies $\operatorname{rank}(M)=|Z|+|T|$, where $|Z|$ and $|T|$ are cardinalities of $Z$ and $T$, respectively.

Proof. Without loss of generality we consider the Lie algebra $L=A *_{a b} F_{n}$, where $A \neq$ $\{0\}$. It is clear that $Y \cup X$ is a minimal generating set and $(Y, X)$ is a minimal generating pair. If $(Z, T)$ is a minimal generating pair of $L$ then by definition, $L=\langle Z\rangle *_{a b}\langle T\rangle$, $\langle Z\rangle$ is a free abelian Lie algebra of rank $|Z|$ and $\langle T\rangle$ is a free Lie algebra of rank $|T|$. Hence by Lemma 2.2, $\operatorname{rank}(L)=|Z|+|T|$.

2.4. Proposition. Every nontrivial subalgebra of $L$ is an abelian product of a free abelian Lie algebra and a free Lie algebra.

Proof. Let $H$ be a subalgebra of $L$. Clearly if $|X|=0,1$ then $L$ is free abelian and so $H$ is free abelian. if $|Y|=0$ then $H$ is a free subalgebra. So the result follows. Assume $|X| \geq 2$. Consider the inclusion map $i: A \rightarrow L$ and the projection $\pi: L \rightarrow F_{n}$. Since for every $a \in A$ and $f \in F_{n}, i(a)=a$ and $\pi(a+f)=f$ we have

$$
\operatorname{Ker} \pi=\operatorname{Imi}=A \text {. }
$$

Restricting $\pi$ to the subalgebra $H$ we get $\{0\} \subseteq K e r \pi_{\mid H}=K e r \pi \cap H=A \cap H \subseteq A$ and $\{0\} \subseteq \pi(H) \subseteq F_{n}$. Therefore $\operatorname{Ker} \pi_{\mid H}$ is a free abelian Lie algebra and $\pi(H)$ is a free Lie algebra. Since $\pi(H)$ is free, $\pi_{\mid H}$ has a splitting $\alpha: \pi(H) \rightarrow H$ sending back each element of a chosen free generating set of $\pi(H)$ to an arbitrary preimage. Hence $\alpha$ is injective and $\alpha \pi(H) \cong \pi(H)$. Every element $h$ of $H$ can be decomposed as $h=h-\alpha \pi(h)+\alpha \pi(h)$. Clearly $h-\alpha \pi(h) \in \operatorname{Ker} \pi_{\mid H}$. Thus

$$
H=K e r \pi_{\mid H} *_{a b} \alpha \pi(H)
$$

is the free abelian product of $\operatorname{Ker} \pi_{\mid H}$ with $\alpha \pi(H)$. This completes the proof.

2.5. Remark. Proposition 2.4 gives us a way of decomposing $H$ into an abelian product of a free abelian subalgebra and a free subalgebra:

$$
H=(H \cap A) *_{a b} \alpha \pi(H)
$$

where $H \cap A$ and $\alpha \pi(H)$ are the free abelian and free parts of $H$, respectively. This decomposition gives a characterization of minimal generating sets and ranks of an arbitrary subalgebra $H$ of $L$.

2.6. Corollary. Let $H$ be a subalgebra of $L$ and $E$ a subset of $H$. Then $E$ is a minimal generating set of $H$ in the sense of Definition 2.1 if and only if $E=E_{A} \cup E_{\alpha \pi(H)}$ or $E$ can be transformed into a set of the form $E_{A} \cup E_{\alpha \pi(H)}$, where $E_{A}$ is a basis of $H \cap A$ and $E_{\alpha \pi(H)}$ is a free generating set of $\alpha \pi(H)$, for a certain splitting $\alpha$ as in the proof of Proposition 2.4.

Proof. By Proposition 2.4, $H$ is a free abelian Lie algebra or a free Lie algebra or it is of the form $H=(H \cap A) *_{a b} \alpha \pi(H)$. Let $E$ be a subset of the subalgebra $H$ of $L$.If $E$ can be transformed into a set of the form $E_{A} \cup E_{\alpha \pi(H)}$ then without loss of generality we can assume that $E=E_{A} \cup E_{\alpha \pi(H)}$, where $E_{A}$ is a basis of $H \cap A$ and $E_{\alpha \pi(H)}$ is a free generating set of $\alpha \pi(H)$.Then $\left(E_{A}, E_{\alpha \pi(H)}\right)$ is a minimal generating pair by Definition 2.1 and $E=E_{A} \cup E_{\alpha \pi(H)}$ is a minimal generating set. Suppose now that $E$ is a minimal generating set of $H$ in the sense of Definition 2.1. Then it is defined by a minimal generating pair $(Z, T)$. Thus $E=Z \cup T$. We now consider the decomposition (2.1) for a choosen splitting $\alpha$. If $\operatorname{rank}(\pi(H))=0$, then $H$ is abelian, $Z$ is an abelian basis of $H$ and $T$ is empty. In this case $H \cap A$ having $Z$ as an abelian basis. Taking $Z=E_{A}$ 
and $T=E_{\alpha \pi(H)}=\emptyset$ leads the result. If $\operatorname{rank}(\pi(H))=1$ then $H$ is again abelian and $Z$ is an abelian basis for $H$. Let $Z=\left\{z_{1}, \ldots, z_{r}\right\}$. Since $\operatorname{rank}(\pi(H))=1$, each $z_{i}$ is in the form $c_{i}+\alpha_{i} w$, where $c_{i}$ is a linear combination of elements of $Y, \alpha_{i} \in K$ and $w \in \pi(H)$. Since $\operatorname{rank}(\pi(H))=1$, at least one of the elements $Z$ is of the form $c_{i}+\alpha_{i} w$ where $\alpha_{i} \neq 0$. If exactly one element in $Z$ is in the form $c_{i}+\alpha_{i} w$,where $\alpha_{i} \neq 0$, then take $E_{\alpha \pi(H)}=\left\{c_{i}+\alpha_{i} w\right\}$ and $E_{A}=Z \backslash\left\{c_{i}+\alpha_{i} w\right\}$. If at least two $\alpha_{i}$ 's nonzero then without loss of generality we may assume that the set $Z$ is in the form $\left\{c_{1}+\alpha_{1} w, \ldots, c_{s}+\alpha_{s} w, c_{s+1}, \ldots, c_{r}\right\}, s \geq 2, \alpha_{i} \neq 0,1 \leq i \leq s$. Applying the transformation $\theta$ defined as

$$
\begin{aligned}
\theta: \quad z_{i} & \rightarrow \alpha_{i+1} z_{i}-\alpha_{i} z_{i+1}, i=1, . ., s-1, \\
z_{j} & \rightarrow z_{j}, j \geq s
\end{aligned}
$$

we can transform the set $Z$ into the form $\left\{a_{1}, \ldots, a_{s-1}, c_{s}+\alpha_{s} w, c_{s+1}, \ldots, c_{r}\right\}$, where $a_{1}, \ldots, a_{s-1}$ are linear combinations of elements of $Y$. Choosing $E_{A}=\left\{a_{1}, \ldots, a_{s-1}, c_{s+1}, \ldots, c_{r}\right\}$ and $E_{\alpha \pi(H)}=\left\{c_{s}+\alpha_{s} w\right\}$, we obtain the result. In the case $\operatorname{rank}(\pi(H))>1$ the restriction $\pi_{\mid<T>}:\langle T>\rightarrow \pi(H)$ is an isomorphism. Now we take the pull back $\alpha$ as $\alpha=\pi_{\mid<T>}^{-1}$. Then $E_{\alpha \pi(H)}$ is a free generating set of $\alpha \pi(H)$. Hence the result follows.

2.7. Corollary. Let $H$ be a subalgebra of $L$ and $(Z, T)$ be a minimal generating pair of

$H$. Then $\operatorname{rank}(H)=|Z|+|T|$, where $0 \leq|Z| \leq m$ and

i) in case of $n=0,1: 0 \leq|T| \leq n$

ii) in case of $n \geq 2: 0 \leq|T| \leq \varkappa_{0}$.

2.8. Corollary. Let $H$ be a subalgebra of $L$. Then $H$ is finitely generated if and only if $\pi(H)$ is finitely generated.

Proof. Let $H$ be a subalgebra of $L$. Consider the decomposition $H=K e r \pi_{\mid H} *_{a b} \alpha \pi(H)$. If $H$ is finitely generated then by Corollary 2.6 it has a finite minimal generating set $E$ which is of the form $E=E_{K e r \pi_{\mid H}} \cup E_{\alpha \pi(H)}$, where $E_{K e r \pi_{\mid H}}$ is a basis of $K e r \pi_{\mid H}$ and $E_{\alpha \pi(H)}$ is a free generating set of $\alpha \pi(H)$. Thus $\alpha \pi(H)$ is finitely generated. Since $\pi(H)$ is isomorphic to $\alpha \pi(H)$ then $\pi(H)$ is also finitely generated.

Now assume that $\pi(H)$ is finitely generated. Any minimal generating set $E$ of $H$ is of the form $E=E_{K e r \pi_{\mid H}} \cup E_{\alpha \pi(H)}$ or it can be transformed into this form, where $E_{K e r \pi_{\mid H}}$ is a generating set of $K e r \pi_{\mid H}$ and $E_{\alpha \pi(H)}$ is a free generating set of $\alpha \pi(H)$. Any subalgebra of a finitely generated free abelian Lie algebra is finitely generated. Using the fact

$$
<E_{K e r \pi_{\mid H}}>=K e r \pi_{\mid H}=H \cap A \subseteq A
$$

we obtain that $E_{K e r \pi_{\mid H}}$ is finite. Therefore $E$ is finite.

2.9. Proposition. Let $L$ be finitely generated and $H$ be a subalgebra of $L$ which is given by a finite set of generators. Then there is an algorithm computing a generating set for $H$ and writing the new and old generators in terms of each other.

Proof. Let $H$ be a subalgebra of $L$ which is given by a finite set of generators $c_{1}+$ $w_{1}, \ldots, c_{p}+w_{p}$, where $p \geq 1, c_{i} \in A, w_{i} \in F_{n}, i=1,2, \ldots, p$. If all $w_{i}$ 's are zero then, $H$ is free abelian and $c_{1}, \ldots, c_{p}$ are generators of $H$. Applying elementary Lie transformations to the set $\left\{c_{1}, \ldots, c_{p}\right\}$, (see[2]), we get a minimal generating set for $H$.

Now assume that $w_{i} \neq 0$ for at least one $i$. We can obtain a free subset $\left\{u_{1}, \ldots, u_{r}\right\}$ of $F_{n}$ by applying suitable elementary Lie transformations to the set $\left\{w_{1}, \ldots, w_{p}\right\}$ (see [7]), where $0 \leq r \leq p$. Clearly $\left\{u_{1}, \ldots, u_{r}\right\}$ is a free generating set of $\pi(H)=<w_{1}, \ldots, w_{p}>$. We have an effective way to express the elements $u_{1}, \ldots, u_{r}$ as words on $w_{1}, \ldots, w_{p}$, say

$$
u_{j}=u_{j}\left(w_{1}, \ldots, w_{p}\right), j=1, \ldots, r
$$


as well as express the elements $w_{1}, \ldots, w_{p}$ in terms of $u_{1}, \ldots, u_{r}$, say

$$
w_{i}=f_{i}\left(u_{1}, \ldots, u_{r}\right), i=1, \ldots, p .
$$

We now consider the map

$$
\alpha: \pi(H) \rightarrow H, \alpha\left(u_{j}\right)=u_{j}\left(c_{1}+w_{1}, \ldots, c_{p}+w_{p}\right), j=1, \ldots, r .
$$

Since

$$
u_{j}\left(c_{1}+w_{1}, \ldots, c_{p}+w_{p}\right)=a_{j}+u_{j}\left(w_{1}, \ldots, w_{p}\right),
$$

where $a_{j}, j=1, \ldots, r$, are linear combinations of $c_{1}, \ldots, c_{p}$, then

$$
\begin{aligned}
\alpha \pi\left(c_{i}+w_{i}\right) & =\alpha\left(w_{i}\right) \\
& =\alpha\left(f_{i}\left(u_{1}, \ldots, u_{r}\right)\right) \\
& =f_{i}\left(a_{1}+u_{1}, \ldots, a_{r}+u_{r}\right) \\
& =d_{i}+f_{i}\left(u_{1}, \ldots, u_{r}\right)
\end{aligned}
$$

where $d_{i}$ is a linear combination of $a_{1}, \ldots, a_{r}, i=1, \ldots, p$. Hence the mapping $\alpha$ can serve as a splitting.

We now determine a generating set for $\operatorname{Ker} \pi / H=A \cap H$. For each given generator $c_{i}+w_{i}$, calculate $c_{i}+w_{i}-\alpha \pi\left(c_{i}+w_{i}\right)$ :

$$
\begin{aligned}
c_{i}+w_{i}-\alpha \pi\left(c_{i}+w_{i}\right) & =c_{i}+w_{i}-d_{i}-f_{i}\left(u_{1}, \ldots, u_{r}\right) \\
& =c_{i}-d_{i}
\end{aligned}
$$

Thus $c_{i}+w_{i}-\alpha \pi\left(c_{i}+w_{i}\right) \in \operatorname{Ker} \pi / H$.

Since $H=K \operatorname{Ker} \pi_{\mid H} *_{a b} \alpha \pi(H)$ then the set $\left\{s_{1}, \ldots, s_{p}\right\}$ generates $\operatorname{Ker} \pi_{\mid H}=H \cap A$, where $s_{i}=c_{i}-d_{i}, i=1, \ldots, p$. Applying elementary transformations to the set $\left\{s_{1}, \ldots, s_{p}\right\}$, we obtain a linearly independent generating set $b_{1}, \ldots, b_{l}$ for $H \cap A$, where $0 \leq l \leq p$.

We get a minimal generating pair $(B, C)$ for $H$ by considering the following cases:

i) If $r=1$ then take $B=\left\{b_{1}, \ldots, b_{l}, a_{1}+u_{1}\right\}$ and $C=\emptyset$,

ii) If $r \neq 1$ then take $B=\left\{b_{1}, \ldots, b_{l}\right\}$ and $C=\left\{a_{1}+u_{1}, \ldots, a_{r}+u_{r}\right\}$

Now we are going to compute the expressions of the new and old generators in terms of each other as the following:

We have

$$
a_{j}+u_{j}=u_{j}\left(c_{1}+w_{1}, \ldots, c_{p}+w_{p}\right), j=1, \ldots, r .
$$

We can also compute expressions of $b_{1}, \ldots, b_{l}$ in terms of $s_{1}, \ldots, s_{r}$ and of the $s_{1}, \ldots, s_{r}$ in terms of the $c_{1}+w_{1}, \ldots, c_{p}+w_{p}$. Therefore we can compute the expressions of the new generators in terms of the old generators.

For the other direction we have

$$
w_{i}=f_{i}\left(u_{1}, \ldots, u_{r}\right), i=1, \ldots, p
$$

Hence

$$
f_{i}\left(a_{1}+u_{1}, \ldots, a_{r}+u_{r}\right)=d_{i}+f_{i}\left(u_{1}, \ldots, u_{r}\right)=d_{i}+w_{i} .
$$

But

$$
c_{i}+w_{i}-\left(d_{i}+w_{i}\right)=c_{i}-d_{i} \in A \cap H .
$$

So we can write the elements $c_{i}-d_{i}$ as

$$
c_{i}-d_{i}=\beta_{1} b_{1}+\ldots+\beta_{l} b_{l}, i=1, \ldots, p .
$$


Thus

$$
\begin{aligned}
c_{i}+w_{i} & =c_{i}-d_{i}+d_{i}+w_{i} \\
& =\beta_{1} b_{1}+\ldots+\beta_{l} b_{l}+d_{i}+w_{i} \\
& =\beta_{1} b_{1}+\ldots+\beta_{l} b_{l}+f_{i}\left(a_{1}+u_{1}, \ldots, a_{r}+u_{r}\right)
\end{aligned}
$$

where $i=1, \ldots, p$.

2.10. Proposition. Given elements $g, h_{1}, \ldots, h_{p} \in L$, it is decidable whether $g \in H=<$ $h_{1}, \ldots, h_{p}>$.

Proof. Let $g \in L$. Write $g=a+w$, where $a \in A, w \in F_{n}$. Let $\left\{b_{1}, \ldots, b_{l}, a_{1}+u_{1}, \ldots, a_{r}+\right.$ $\left.u_{r}\right\}$ be a generating set of $H$. Now check whether $\pi(g)=w \in \pi(H)=<u_{1}, \ldots, u_{r}>$. We can derive from [1] that the subalgebra membership problem is decidable for free Lie algebras. Since $\pi(H)$ is a free Lie algebra we can decide whether $\pi(g) \in \pi(H)$. Now if $\pi(g) \notin \pi(H)$, then $g \notin H$. If $\pi(g) \in \pi(H)$, then $\pi(g)=w$ can be expressed in terms of free generators $u_{1}, \ldots, u_{r}$, say $w=f\left(u_{1}, \ldots, u_{r}\right)$. Computing $f\left(a_{1}+u_{1}, \ldots, a_{r}+u_{r}\right)$ we get

$$
f\left(a_{1}+u_{1}, \ldots, a_{r}+u_{r}\right)=c+f\left(u_{1}, \ldots, u_{r}\right)=c+w \in H .
$$

It is clear that

$$
g=a+w \in H \text { if and only if } a-c=a+w-(c+w) \in H,
$$

that is

$$
g=a+w \in H \text { if and only if } a-c \in<b_{1}, \ldots, b_{l}>\subseteq A .
$$

In the affirmative case we can compute $g$ in terms of the elements of the set $\left\{b_{1}, \ldots, b_{l}, a_{1}+\right.$ $\left.u_{1}, \ldots, a_{r}+u_{r}\right\}$. Using expressions we already have for the elements of the set $\left\{b_{1}, \ldots, b_{l}, a_{1}+\right.$ $\left.u_{1}, \ldots, a_{r}+u_{r}\right\}$ in terms of the $h_{i}$ 's we find an expression for $g$ in terms of the $h_{i}$ 's.

2.11. Remark. Let $H$ be a subalgebra of $L$ and $(B, C)$ be a minimal generating pair of $H$, where $B=\left\{b_{1}, \ldots, b_{l}\right\}, C=\left\{a_{1}+u_{1}, \ldots, a_{r}+u_{r}\right\}, 0 \leq l \leq m, a_{i} \in A, u_{i} \in F_{n}, i=$ $1, \ldots, r$. Then $\left\{u_{1}, \ldots, u_{r}\right\}$ is a free generating set of $\pi(H)$ (see the proof of Proposition $2.9)$ and $B$ is an abelian basis of $H \cap A$. Every element $h$ of $H$ can be written as

$$
h=\sum_{i=1}^{l} \beta_{i} b_{i}+\sum_{i=1}^{r} \gamma_{i} a_{i}+w\left(u_{1}, \ldots, u_{r}\right),
$$

where $\beta_{i}, \gamma_{i} \in K, 1 \leq i \leq l, 1 \leq j \leq r$ and $\gamma_{i}$ 's are defined uniquely by $w$. We can write the elements $a_{i} \in A$ as

$$
a_{i}=\sum_{j=1}^{m} \delta_{i j} y_{j}, \delta_{i j} \in K, i=1, \ldots, r .
$$

Denote by $\overline{a_{i}}$ the vector $\left(\delta_{i 1}, \ldots, \delta_{i m}\right)$. Let $R=\left(\gamma_{1}, \ldots, \gamma_{r}\right), S=\left(\begin{array}{c}y_{1} \\ \cdot \\ \cdot \\ y_{m}\end{array}\right)$ and let $M$ be the $r \times m$ matrix

$$
M=\left(\begin{array}{c}
\overline{a_{1}} \\
\cdot \\
\cdot \\
\cdot \\
\overline{a_{r}}
\end{array}\right)
$$


Then straightforward calculations show that the element $h$ is in the form

$$
h=a+w\left(u_{1}, \ldots, u_{r}\right)
$$

where $w\left(u_{1}, \ldots, u_{r}\right) \in F_{r}$ and $a \in R M S+<B>$ (here $w \in F_{r}$ and $w\left(u_{1}, \ldots, u_{r}\right)$ is the element of $\pi(H)$ obtained by replacing the $i$ th letter in $w$ by $\left.u_{i}, i=1, \ldots, r\right)$.

We have proved the following.

2.12. Lemma. With previous notations, we have

$$
H=\left\{a+w\left(u_{1}, \ldots, u_{r}\right): w\left(u_{1}, \ldots, u_{r}\right) \in \pi(H), a \in R M S+<B>\right\} .
$$

2.13. Definition. Let $H$ be a subalgebra of $L$ and $w \in F_{n}$. The set

$$
C=\{a: a+w \in H\} \subseteq A
$$

is called the abelian completion of $w$.

2.14. Corollary. With the above notation, for every $w \in F_{n}$ we have

i) If $w \notin \pi(H)$, then $C=\emptyset$,

ii) If $w \in \pi(H)$, then $C=R M S+<B>$.

\section{References}

[1] Bahturin Yu., Olshanski A., Filtrations and Distortion in infinite dimensional algebras, arxiv:1002.0015v1[math.RA](29 January 2010)62pp.

[2] Bokut L.A., Kukin G.P., Algorithmic and Combinatorial Algebra, Kluwer Academic Publ.,Dordrecht, 1994.

[3] Delgado J.,Ventura E., Algorithmic problems for free abelian times free group, J. Algebra. (2013)http://dx.doi.org.//101016/J.Algebra.2013.04.033.

[4] Ekici N., Ögüuslü N.Ş., Test rank of an abelian product of a free Lie algebra and a free abelian Lie algebra, Proc. Indian Acad. Sci. (Math. Sci.) 121,3,291-300,2011.

[5] Gupta C.K., Timoshenko E.I., Test rank of a Soluble product of free abelian groups, Sbornik Math. 199,4,495-510,2008.

[6] Gupta C.K., Timoshenko E.I., Criterion for invertibility of endomorphisms and test rank of metabelian product of abelian groups, Alg. Logic,43,5,565-581,2003.

[7] Kukin, G.P., Primitive elements of free Lie algebras, Algebrai Logika 9, 4,458-472,1970. 
